Documentation et bibliothèques

DOCUMENTATION BIBLIOTHEQUES

\title{
La télédocumentation, ou toute la mémoire du monde à domicile
}

\section{Louis Brunel}

Volume 21, numéro 4, décembre 1975

URI : https://id.erudit.org/iderudit/1055379ar

DOI : https://doi.org/10.7202/1055379ar

Aller au sommaire du numéro

\section{Éditeur(s)}

Association pour l'avancement des sciences et des techniques de la documentation (ASTED)

\section{ISSN}

0315-2340 (imprimé)

2291-8949 (numérique)

Découvrir la revue

\section{Citer cet article}

Brunel, L. (1975). La télédocumentation, ou toute la mémoire du monde à domicile. Documentation et bibliothèques, 21(4), 179-186.

https://doi.org/10.7202/1055379ar
Résumé de l'article

L'ère scientifique dans laquelle nous vivons engendre une explosion documentaire qui crée un besoin croissant de systèmes pour faciliter le repérage de l’information et la rendre accessible. La technologie, grâce à son développement accéléré, pourra sans doute, par la création de banques de données et de réseaux d'échanges décentralisés, accélérer l'avènement de la télédocumentation et aider à solutionner les problèmes documentaires.
Tous droits réservés $@$ Association pour l'avancement des sciences et des techniques de la documentation (ASTED), 1975
Ce document est protégé par la loi sur le droit d'auteur. L'utilisation des services d'Érudit (y compris la reproduction) est assujettie à sa politique d'utilisation que vous pouvez consulter en ligne.

https://apropos.erudit.org/fr/usagers/politique-dutilisation/ 


\title{
La télédocumentation, ou toute la mémoire du monde à domicile*
}

\author{
Louls Brunel \\ Vice-président aux Communications \\ Université du Québec \\ Québec
}

L'ère scientifique dans laquelle nous vivons engendre une explosion documentaire qui crée un besoin croissant de systèmes pour faciliter le repérage de l'information et la rendre accessible. La technologie, grâce à son développement accéléré, pourra sans doute, par la création de banques de données et de réseaux d'échanges décentralisés, accélérer l'avènement de la télédocumentation et aider à solutionner les problèmes documentaires.

The scientific age in which we live gives rise to a document explosion and in turn an increasing need for better systems in tracing information and making it available. Owing to its rapid development, technology will undoubtedly speed up the advent of teledocumentation and help to solve documentary problems, through the establishment of data banks and decentralized exchange channels.

Vivimos en una era cientifica que está causando una explosión de documentarios, y la necesidad de sistemas para facilitar la buscada y el encuentro de información está creciendo. Por su desarrollo acelerado, la tecnologia podrá sin duda, con la creación de bancos de información y redes de intercambio descentralizadas, acelerar la llegada de la teledocumentación y ayudar a solucionar los problemas documentarios.

Nous pouvons constater présentement que, si le développement des systèmes de documentation comporte de grands avantages, l'encombrement qui en résulte nous place néanmoins devant la menace croissante de l'asphyxie.

\section{L'ère scientifique}

La société de l'information, résultante de l'ère post-industrielle, est le fruit d'une longue évolution des civilisations et du déplacement de leurs forces composantes.

Henri Prat a bien situé, il y a déjà quinze ans, l'ère scientifique dans l'évolution humaine lorsqu'il a écrit:

"Qu'en raison même de notre entrée dans l'ère scientifique, la puissance réelle, celle qui peut infléchir le cours des événements, change de mains progressivement. Dans les sociétés agricoles de type féodal, elle appartenait au seul guerrier. Avec la création des États organisés, elle passa à l'homme politique. Avec l'avènement 
du machinisme, au XIXe siècle, ce furent les forces industrielles qui s'en emparèrent peu à peu. Aujourd'hui, qu'on le veuille ou non, elle échoit pour une part sans cesse croissante aux scientifiques, aux techniciens de tous ordres...

...ll s'agit là d'un simple fait d'évidence: que les sciences pures et appliquées, les techniques de tous ordres, prennent nécessairement une place sans cesse croissante dans la vie de toutes les collectivités».'

\section{L'explosion documentaire}

Mais ce "simple fait d'évidence» n'est pas sans conséquence. Une étude récente de l'Organisation de coopération et de développement économiques (OCDE) portant sur l'information dans une société en évolution tire la conclusion suivante:

"La science et la technique se sont révélées un bienfait pour l'humanité et sont essentielles à son avenir, mais il ne faut pas se leurrer sur toutes les conséquences qu'elles peuvent avoir. Face à l'expansion rapide du savoir, il est de plus en plus difficile de partager et d'utiliser les informations avec fruit. Les méthodes classiques sont trop lentes et trop coûteuses; en outre, elles ne permettent pas de procéder à l'évaluation et à la sélection qu'exige toute utilisation fructueuse". ${ }^{2}$

Le développement spectaculaire et accéléré des découvertes et des connaissances scientifiques engendre une accumulation de documentation et un encombrement d'informations sans précédent. Depuis quelques décennies, la masse documentaire s'accroît, de fait, à un rythme exponentiel, si bien qu'on assiste actuellement à une véritable explosion de la documentation.

Selon différentes sources ${ }^{3}$ on évalue la production annuelle d'écrits scientifiques de toutes sortes à 2 millions, soit un demi-milliard de pages; chaque année quelque 100,000 revues sont publiées et font l'objet de plus d'un million et demi de "condensés", que diffusent à leur tour plus de 1,800 services spécialisés dans la description bibliographique.

Ces chiffres ne surprennent guère lorsque l'on sait que les seuls services scientifiques et techniques du gouvernement fédéral des États-Unis diffusent annuellement quelque 80,000 rapports techniques.

Cette volumineuse production courante vient s'ajouter à un stock mondial d'environ 25 à 30 millions de titres d'articles scientifiques et techniques, totalisant 10 mille milliards de caractères alphanumériques. ${ }^{+}$

1. Henri Prat, Métamorphose explosive de l'humanité, Paris, 1961, II, p. 163-164.

2. Pierre Piganiol, L'information dans une société en évolution. Paris, OCDE, 1971, p. 45.

3. Ferdinand F. Leimkuhler et Anthony E. Neville, "The uncertain future of the library", The John Hopkins Magazine, (Autumn 1967), 15; W.L. Saunders, "Economic success: the contribution of the information scientist". The Information Scientist, vol. 2, no. 3 (1969), 118-119; Georges Anderla, "L'avenir de l'information: un défi pour les gouvernements et la société". L'observateur de l'OCDE, no 63 (avril 1973), 27-33. 
De plus, les prévisions pour un futur très proche ne semblent pas vouloir nous rassurer puisque l'on estime que le nombre de documents scientifiques mis en circulation pendant la seule année 1985, sera de l'ordre de huit à dix millions, soit un volume de quelque quatre à cinq fois plus considérable que celui dont nous venons de parler.

Cet encombrement est illustré encore par la tendance des bibliothèques vers le gigantisme: la Bibliothèque nationale de $P$ aris renferme près de 16 millions d'unités documentaires; ; la Bibliothèque Lénine de Moscou contient près de 28 millions d'ouvrages ${ }^{6}$; la Library of Congress de Washington qui comptait, pour sa part, près de 33 millions de documents en 1955, en a maintenant quelque 72 millions?

\section{La documentation automatique}

Cette avalanche de chiffres nous permet d'illustrer l'explosion documentaire que l'ère scientifique produit. A cette explosion documentaire correspond le recours de plus en plus répandu à l'informatique pour la signalisation, le repérage et la gestion des documents. Ainsi, les fiches cartonnées habituelles des fichiers sont remplacées par des bandes ou disques magnétiques reliés à des ordinateurs et dont le contenu est accessible, à l'aide de terminaux, à l'utilisateur.

Cette fascinante réalisation qui nous ouvre la possibilité d'interroger à distance des banques de données grâce aux réseaux de télécommunications a déjà atteint un niveau d'activités important puisqu'il existe plus de 100 banques de données commerciales dans le seul domaine de la science et de la technologie. L'accroissement annuel du nombre d'enregistrements dans ces banques est de l'ordre de trois millions. En particulier, la banque de données de l'Institute for Scientific Information (ISI), qui utilise l'indexage des citations, connaît à elle seule un rythme d'accroissement de près de 400,000 documents par année qui représentent 4 millions de citations. On peut raisonnablement estimer que les enregistrements bibliographiques, pour leur part, s'accroissent annuellement au rythme de 625 millions de caractères alphanumériques alors que les enregistrements de résumés («abstracts») augmentent de 375 millions par an.

C'est donc dire que l'accroissement annuel du nombre d'enregistrements dans les banques de données spécialisées dans le domaine de l'information scientifique et technique atteint déjà le milliard de caractères alphanumériques! Même si l'on pose comme hypothèse que l'accroissement significatif n'est que de l'ordre du demi-milliard à cause de la duplication des enregistrements dans les différentes banques de données, il n'est certes pas abusif de parler d'encombrement.

Une conséquence évidente de cet encombrement, c'est qu'il devient de plus en plus difficile de se retrouver dans cette masse documentaire.

Autre conséquence aussi troublante, c'est que la documentation devient de plus en plus éphémère, réduisant ainsi d'autant la période de vie utile du document. II est à peine exagéré de dire qu'au moment de la parution d'un livre, son contenu est déjà périmé. Les délais de publi-

5. World of Learning, 1973-74, p. 415.

6. Ibid., 1346.

7. Change, (April 1975), 48. 
cation sont tels que certains organismes scientifiques publient des listes où sont décrits, avant leur parution, les articles scientifiques à venir.

\section{L’approche systémique}

Cette explosion de l'information sous toutes ses formes engendre un besoin croissant de systèmes qui permettent la collecte, le repérage et le traitement de l'information et en garantissent l'accessibilité.

Les divers vocabulaires utilisés pour le traitement de la documentation visent à classer et répertorier des ensembles documentaires de plus en plus vastes de façon à assurer un repérage et une diffusion adéquats. "Grâce à l'ordinateur". écrit Gérard Mercure, chercheur en télédocumentation, "il n'est plus maintenant seulement question de diffusion, mais de diffusion sélective, selon un profil d'intérêts correspondant au champ d'activité d'un chercheur ou d'un groupe de chercheurs" ${ }^{8}$

La création de banques de documentation exige la conception de systèmes complexes dont la mise au point requiert l'apport conjugué d'une foule de spécialistes: bibliothécaires, informaticiens, linguistes, informatistes, pour n'en nommer que quelques-uns, car il est de plus en plus évident, comme le mentionnait le rapport Whitehead que:

«...dans un univers dont la complexité ne fait qu'augmenter nous sommes amenés à penser de plus en plus en termes d'un concept global de l'information, en particulier si nous voulons infléchir le progrès en fonction de nos objectifs plutôt que de seulement réagir au changement." ${ }^{9}$

Une fois ces systèmes développés, il faut en assurer l'implantation et prévoir des normes d'exploitation qui permettront de satisfaire les utilisateurs; il faut, de plus, garantir la qualité du contenu des banques de données ainsi que leur fiabilité pour permettre une utilisation optimale des ressources et une accessibilité maximale à l'information.

Le jumelage des techniques de la microcopie et des télécommunications contribue déjà à solutionner les problèmes de l'accessibilité à la documentation; on peut maintenant envisager l'utilisation généralisée de bélinographes permettant de transmettre des "fac-similés" et des microcopies aux utilisateurs, quel que soit l'endroit où ils se trouvent.

L'ère des systèmes statiques de documentation est révolue; ce n'est plus l'utilisateur qui va à l'information, mais c'est l'information qui rejoint l'usager. Nous passons de l'époque du guichet à celle du libre-service. Les procédés artisanaux de transmission de l'information par le livre feront place à une véritable industrie du savoir qui caractérisera la prochaine décennie.

On affirme, à ce sujet, dans une étude de l'OCDE portant sur l'information que:

8. Gérard Mercure, "Le mur de l'imprimé ", Bulletin de l'ACBLF, vol. XVIII, no 3 (septembre 1972), 160.

9. J.R. Whitehead, Report of the ad hoc Group on Information, Computers and Communications. Document SP (71) 19, 25 juin 1971, Paris, OCDE, p. 7. La traduction est de l'auteur. 
"Selon l'opinion collective de plusieurs centaines de savants et d'experts, l'avenir de l'industrie du savoir et celui de toutes ses composantes sans exception sont étroitement liés à l'automatisation de l'information. De l'avis unanime, l'information automatisée supplantera définitivement, au cours de la décennie 1980-1990, les procédés artisanaux qui assument actuellement, tant bien que mal, la transmission et la diffusion des connaissances.

C'est alors seulement que l'information sera devenue, pour les diverses branches de l'industrie du savoir, un véritable input, précieux et universellement apprécié, et pour les hommes qui s'y emploient, un véritable outil de travail d'une efficacité incomparable.

Telles sont les principales conclusions qui se dégagent avec beaucoup de netteté de toutes les enquêtes Delphi.» ${ }^{10}$

\section{Le développement de la technologie}

Mais ces nouveaux services de l'ère de l'information à demande ne seront-ils pas eux-mêmes des facteurs d'encombrement et ne risquentils pas de contribuer ainsi à leur propre paralysie?

Nous sommes d'avis que le rythme exponentiel du développement de la technologie permet d'envisager des solutions de désencombrement fort efficaces en offrant, par exemple, des supports de stockage de grande capacité, ultra-rapides, de taille microscopique et à des coûts réalistes.

Grâce à des enquêtes Delphi, on a établi le moment historique où la majorité des bibliothèques et des centres de documentation, de caractère scientifique et technique, seront réorganisés et à peu près entièrement automatisés entre 1985 et 1990 . On prévoit qu'alors ils assumeront un véritable libre-service documentaire, toute information demandée par n'importe quel utilisateur étant automatiquement extraite de mémoires de masse ultra-rapides, projetée sur écran cathodique et imprimée sur demande.

II est même prévu que, dès cette période, le public aura, au même titre que les spécialistes, accès aux centres d'information par le truchement de réseaux de câblodistribution.

Ces prévisions sur l'utilisation future de la technologie pour les fins des systèmes documentaires ne doivent pas nous surprendre car les spécialistes de la Mitre Corporation prévoient qu'au cours de la présente décennie le nombre de systèmes intégrant ordinateurs et télécommunications sera multiplié par un facteur de 200.

Une étude de Dieter Kimbel ${ }^{11}$ prévoit également que, pour la même période, dans le seul secteur des services d'information, le nombre de points terminaux desservis passera de 32,000 à un demi-million; le nombre de terminaux installés, de 45,000 à 800,000 ; et le nombre de transactions à distance, de 600 millions à quelque 60 milliards d'unités.

Le groupe Diebold estime, pour sa part, que le nombre total de terminaux aux États-Unis devrait progresser de 30,000, en 1965, à 2 mil-

10. Georges Anderla, L'information en 1985, Paris, OCDE, 1971, p. 73.

11. Dieter Kimbel, Computers and Telecommunications, Paris, OCDE, 1973. 
lions à la fin de l'année 1975, leur valeur marchande passant de 100 millions à 5 milliards et demi de dollars. ${ }^{12}$ Enfin, selon la Datapro Research Corporation, alors que 700,000 terminaux étaient en opération aux États-Unis en 1972, on en prévoit 7 millions pour $1982 .{ }^{13}$

Toutes ces données illustrent bien que, si l'explosion de la documentation se produit à un rythme exponentiel, le développement technologique, pour sa part, est caractérisé par une croissance encore plus accélérée. En fait, le développement de la technologie s'accélère à un rythme qui est plus de deux fois plus rapide que le taux d'expansion de l'explosion documentaire.

\section{La technologie compétitive}

Mais par quel enchaînement parviendrons-nous à cette société de l'information? Quel levier pourra nous permettre de passer de l'indice 1 à l'indice 50 ou 100 ?

II existe, bien sûr, des facteurs d'innovation et des raisons de prestige, de sécurité ou de contrôle qui sont à l'origine de l'implantation de systèmes de documentation automatique; mais la condition essentielle d'une exploitation généralisée de tels systèmes est qu'il devient économiquement plus avantageux d'assurer l'accès à la documentation par des moyens électroniques plutôt que par des procédés manuels.

Sous cet aspect également, la capacité et la performance de la technologie croissent à un rythme nettement plus rapide que celui de l'augmentation de la masse documentaire. En conséquence, les coûts unitaires décroissent aussi de façon exponentielle.

Ainsi, en 1945, un million d'opérations arithmétiques effectuées sur une machine à calculer électrique, revenait à quelque $i, 000$ dollars. La première génération d'ordinateurs a permis d'abaisser ce prix de revient à 10 dollars. Dix ans plus tard, l'exécution de la même tâche ne coûtait plus que cinq cents et, aujourd'hui, la dépense est de l'ordre d'un demi-cent. De 1,000 dollars à un demi-cent, cela représente un indice de réduction de 200,000 en 30 ans!

Les mémoires de masse, pour leur part, introduisent également des changements considérables dans l'économie générale de la technologie. Il en existe actuellement, et nous n'en sommes qu'à la première génération, qui ont une capacité de mille milliards de "bits" d'information. Le coût de stockage se situe à environ deux dollars par mois par million de caractères. A titre indicatif, les unités de disques dont nous disposons à l'heure actuelle contiennent 100 millions de caractères à un coût de six à sept dollars par mois par million de caractères. Cette capacité sera accrue par un facteur de 1.000 grâce aux mémoires de masse et le prix de revient sera alors réduit au tiers.

Telles sont les économies d'échelle que le progrès technologique laisse entrevoir. 


\section{Les réseaux}

Le résultat de l'explosion de la documentation et de l'accélération vertigineuse du progrès technologique sera-t-il néfaste à leur utilisateur, l'homme?

Dans ces développements vertigineux, saurons-nous conserver une dimension humaine à nos réalisations? Car - et il faut en être conscient - pour être utiles et efficaces, les systèmes d'information devront toujours être en rapport étroit avec leurs utilisateurs; les données fournies seront alors adaptées, conformément aux objectifs de l'industrie du savoir, aux besoins de la clientèle, et répondront vraiment à leurs exigences.

Malgré ces exigences du marketing, nombreux sont ceux qui, devant les possibilités mirobolantes de la technologie, plaident en faveur de systèmes hautement centralisés; l'efficacité de tels systèmes nous paraît problématique si une certaine taille critique est dépassée. Même si la centralisation de tels systèmes comporte des avantages, elle oblige à gérer de gigantesques banques d'information, exige des investissements énormes et risque de ne pas présenter suffisamment de souplesse face aux besoins variables des utilisateurs. Ces systèmes, à cause de leur rigidité, sont menacés, à brève échéance, de paralysie.

II ne fait aucun doute que le système d'information de demain se présentera plutôt sous la forme d'un ensemble de sous-systèmes spécialisés, interconnectés entre eux par des réseaux de télécommunications très évolués. Ce système global permettra ainsi de transmettre, d'un sous-système à un autre, les demandes auxquelles un sous-système donné ne pourra répondre; il assurera la satisfaction des demandes spécifiques des utilisateurs grâce à des adaptations particulières rendues possibles par l'échelle "humaine" de chaque sous-système. Le réseau américain ARPA, qui relie un grand nombre d'ordinateurs universitaires, a été développé selon cette philosophie et a été un précurseur dans cette voie.

Le réseau de l'Université du Québec s'est développé dans ce sens. II est constitué d'un ordinateur principal Control Data de la série CYBER, de trois ordinateurs satellites, d'une douzaine de terminaux lourds et de plus d'une centaine de terminaux lents. L'ordinateur principal du réseau est installé sur le campus de l'Université Laval, dans des locaux adjacents à ceux du Centre de traitement de l'information de l'Université Laval.

Cette situation privilégiée permet l'accès dans tout le réseau de l'Université du Quẹbec au langage APL de I'Université Laval.

De plus, les utilisateurs du réseau ont maintenant accès directement au système de recherche documentaire Canadian On-Line Enquiry (CAN/OLE), exploité à Ottawa par l'Institut canadien d'information scientifique et technique du Conseil national de recherches du Canada. L'accès à cette banque de données élargit l'éventail des moyens offerts aux partenaires du réseau, en leur signalant des documents provenant de sources qu'ils n'ont pas l'occasion de consulter. D'ici quelques mois, ils auront également accès aux banques de données de System Development Corporation et de Lockheed.

De plus, le réseau de l'Université du Québec fournit à ses partenaires un système de repérage bibliographique appelé BADADUQ (Banque de données à accès direct de l'Université du Québec). Ce 
système offre un large éventail de descripteurs. Le vocabulaire est libre et les usagers peuvent ajouter des descripteurs.

\section{La télédocumentation}

II y aura d'autres étapes dans le développement de la télédocumentation, entre autres la lecture courante et l'analyse systématique de documents par ordinateur, la "télécirculation", le livre télédiffusé et le vidéophone, l'objectif ultime étant toujours de permettre l'accès à distance aux sources du savoir.

Si nous parlons de "sources du savoir", c'est par opposition à ce qu'on pourrait appeler les "cimetières du savoir». Car, pour nous, ce n'est pas le document inerte qui est véritablement à l'origine de la connaissance, de l'information, mais plutôt l'homme de science, le chercheur. En ce sens, on peut dire que l'objectif ultime de la télédocumentation est de créer un lien vital entre l'utilisateur et les créateurs de connaissances.

Cet aboutissement sera le fruit d'une longue évolution dont on peut résumer le cheminement de la façon suivante: des informations de plus en plus nombreuses se créent à tout instant. Elles circulent de plus en plus vite. Elles ont une durée de vie utile de plus en plus courte. Elles s'accumulent à une cadence qui, loin de connaître le moindre répit, ne fait que s'accentuer. Gigantisme des bibliothèques, développement vertigineux de banques de données, mise en place de réseaux d'échanges, ce sont là les tendances actuelles du développement de la documentation.

Devant cette marée qui menace de nous submerger, il est possible, pendant quelque temps encore, de tergiverser, de chercher des échappatoires, de se contenter de palliatifs. Mais à la fin de la présente décennie, le choix sera inéluctable: l'asphyxie ou la télédocumentation. A ceux qui demeurent sceptiques devant les perspectives d'avenir de la télédocumentation, nous répondrons avec Jules Verne que "tout ce qu'un homme est capable d'imaginer, d'autres hommes seront capables de le réaliser un jour".

\section{ERRATA:}

Dans la livraison de septembre 1975 de Documentation et bibliothèques, deux références ont été supprimées par erreur, à la fin de l'article de Marielle Durand (p. 120):

13. Saul W. Gellerman, Les motivations, clef d'une gestion efficace, Paris, Dunod, 1971, p. 134.

14. Ibid., 138. 